\title{
Pacific
}

Journal of

Mathematics

\section{SHARING VALUES AND A PROBLEM DUE TO C. C. YANG}

XIN-Hou HuA 


\title{
SHARING VALUES AND A PROBLEM DUE TO C.C. YANG
}

\author{
XIN-HOU HUA
}

In this paper, we proved a unicity theorem for meromorphic functions with one sharing pair and a condition on deficiency. An example shows that the condition on deficiency is best possible. This result gives a general answer to the problem due to C.C.Yang (1977).

\section{Introduction.}

In this paper, by meromorphic function we always mean a function which meromorphic in the plane. Let $f(z)$ be meromorphic. We shall use the following standard notations in Nevanlinna theory:

$$
T(r, f), \quad m(r, f), \quad N(r, f), \ldots
$$

(see Gross [5]). We denote by $S(r, f)$ any function satisfying

$$
S(r, f)=\circ\{T(r, f)\}
$$

as $r \rightarrow+\infty$, possibly outside a set of finite Lebesgue measure. A meromorphic function $a(z)$ is said to be a small function of $f$ if

$$
T(r, a)=S(r, f) .
$$

In this case, we define

$$
\delta(a, f)=1-\varlimsup_{r \rightarrow \infty} \frac{N\left(r, \frac{1}{f-a}\right)}{T(r, f)},
$$

and $a(z)$ is said to be a deficient function of $f$ if $\delta(a, f)>0$.

Let $g(z), a_{1}(z)$ and $a_{2}(z)$ be meromorphic functions. If the two functions $f(z)-a_{1}(z)$ and $g(z)-a_{2}(z)$ assume the same zeros with the same multiplicities, then we call that $f$ and $g$ share the pair $\left(a_{1}, a_{2}\right) \mathrm{CM}$. In particular, if $a_{1}=a_{2}=a$, then the word "the pair" is replaced by "the value" or "the function" provided that $a$ is a constant or $a$ is a function respectively (cf. Frank-Ohlenroth [4], Gundersen [6], etc.). In addition, if

$$
N\left(r,\left(f=a_{1}\right) \Delta\left(g=a_{2}\right)\right)=\min \{S(r, f), \quad S(r, g)\},
$$


then we say that $f$ and $g$ almost share the pair $\left(a_{1}, a_{2}\right)$ CM. Here, $N(r,(f=$ $\left.\left.a_{1}\right) \Delta\left(g=a_{2}\right)\right)$ is the counting function of those points which satisfy one of the following three cases: (i) $f=a_{1}$ but $g \neq a_{2}$; (ii) $f \neq a_{1}$ but $g=a_{2}$; (iii) $f=a_{1}$ and $g=a_{2}$ but the multiplicities are not the same.

In 1977, Yang [9] proved the following result.

Theorem A. Suppose that $F$ is a family of the functions which are of the form $\alpha_{1}(z) e^{\mu(z)}+\alpha_{2}(z)$, where $\mu(z)$ is an entire functions with finite order, $\alpha_{j}(z)(j=1,2)$ are meromorphic functions of finite order, $\alpha_{1} \not \equiv 0$, $\alpha_{2} \not \equiv$ const., the order of $\alpha_{j}(j=1,2)$ is less than the order of $\mu$. Let $c_{1}$ and $c_{2}$ be two distinct constants, and let $f \in F, g \in F$. If $f$ and $g$ share the two values $c_{1}$ and $c_{2} C M$, then $f \equiv g$ or

$$
\left(f-\frac{c_{2}-c_{1} \lambda(z)}{1-\lambda(z)}\right)\left(g+\frac{c_{2}-c_{1} \lambda(z)}{1-\lambda(z)}\right)=-\frac{\left(c_{2}-c_{1}\right)^{2} \lambda(z)}{(1-\lambda(z))^{2}}
$$

where $\lambda(z)$ is a nonconstant meromorphic function.

Based on this result, Yang [9] proposed the following problem.

Yang's problem. Whether can we omit the restrictions on the order in the family $F$ ?

It is easy to see from the hypotheses of Theorem A that, if $f=\alpha_{1}(z) e^{\mu(z)}+$ $\alpha_{2}(z) \in F$ and $g=\alpha_{3}(z) e^{\nu(z)}+\alpha_{4}(z) \in F$, then $N\left(r, \frac{1}{f-\alpha_{2}}\right)=\circ\{T(r, f)\}$ and $N\left(r, \frac{1}{g-\alpha_{4}}\right)=\circ\{T(r, g)\}$. Thus

$$
\begin{aligned}
& \delta\left(\alpha_{2}, f\right)=\delta\left(\alpha_{4}, g\right)=1 \\
& \delta(\infty, f)=\delta(\infty, f)=1 .
\end{aligned}
$$

These observations lead to our main result.

Theorem 1. Let $f(z), g(z), a(z), b(z), \alpha(z)$ and $\beta(z)$ be meromorphic functions in the plane, where $a(z)$ and $\alpha(z)$ are small functions of $f, b(z)$ and $\beta(z)$ are small functions of $g(z), a(z) \not \equiv \alpha(z), b(z) \not \equiv \beta(z)$. Suppose that $f$ and $g$ share the pair $(a, b) C M$ and

$$
\delta=\delta(\alpha, f)+\delta(\beta, g)+\delta(\infty, f)+\delta(\infty, g)>3 .
$$

Then either

$$
\frac{f-\alpha}{a-\alpha}=\frac{g-\beta}{b-\beta}
$$


or

$$
\frac{f-\alpha}{a-\alpha} \frac{g-\beta}{b-\beta}=1
$$

Remark 1. The number 3 in the inequality (3) is sharp.

For example, let $P$ and $Q$ be two nonzero polynomials, $f=e^{2 z}-Q e^{z}$, $g=\frac{e^{2 z}}{e^{z}+\frac{P}{Q}}$. Then one can check that $f$ and $g$ share the pair $(P, Q) \mathrm{CM}$ and

$$
\delta(0, f)+\delta(0, g)+\delta(\infty, f)+\delta(\infty, g)=\frac{1}{2}+1+1+\frac{1}{2}=3 .
$$

However, $\frac{f}{P} \not \equiv \frac{g}{Q}$ and $\frac{f}{P} \frac{g}{Q} \not \equiv 1$.

Remark 2. Note that Theorem A needs two shared values. However, in our theorem 1 , we only need one shared pair.

Remark 3. The topic on unicity theorem concerning deficiency were studied by Ozawa [7], Ueda [8] etc. The case that $f$ and $g$ are entire functions and $a(z)=b(z)=1$ was considered by Yi $[\mathbf{1 0}]$.

Remark 4. From the proof of Theorem 1 we see that the word "share" can be replaced by "almost share".

As an application, we obtain the following

Corollary. The answer to Yang's problem is affirmative.

\section{Some Symbols.}

For the sake of convienence, we shall use some symbols introduced by Chuang [1] and Chuang-Hua [2].

For meromorphic function $f(z)$ and a point $z$, according as $z$ is a pole of f or not, we denote by $\omega(f, z)$ the multiplicity of $z$ or 0 and by $\bar{\omega}(f, z)$ the value 1 or 0 . For three meromorphic functions $f, g$ and $h$, we divide the set of the poles of $f$ and $g$ on $\{|z| \leq r\}$ into five pairwise disjoint subsets as follows:

$$
\begin{array}{lll}
V_{1}=: & \{z: \quad f(z) \neq \infty, \quad g(z)=\infty\}, \\
V_{2}=: \quad\{z: \quad f(z)=\infty, \quad g(z) \neq \infty\}, \\
V_{3}=: \quad\{z: \quad f(z)=\infty, \quad g(z)=\infty, \quad h(z)=\infty\}, \\
V_{4}=: \quad\{z: \quad f(z)=\infty, \quad g(z)=\infty, \quad h(z) \neq 0, \infty\}, \\
V_{5}=: \quad\{z: \quad f(z)=\infty, \quad g(z)=\infty, \quad h(z)=0\} .
\end{array}
$$


Furthermore, for each $j \in\{1, \ldots, 5\}$, we denote by $n_{j}(f)$ and $n_{j}(g)$ the number of the poles of $f$ and $g$ in the set $V_{j}$ respectively, with due count of multiplicity. The corresponding counting functions are denoted by $N_{j}(f)$ and $N_{j}(g)$ respectively. Obviously,

$$
\begin{aligned}
& N(r, f)=N_{2}(f)+N_{3}(f)+N_{4}(f)+N_{5}(f), \\
& N(r, g)=N_{1}(g)+N_{3}(g)+N_{4}(g)+N_{5}(g) .
\end{aligned}
$$

\section{One Basic Lemma.}

For the proof of our results, we need the following lemma which can be found in Chuang-Yang [3, p. 39] or Gross [5, pp. 70-73].

Lemma 1. Let $f_{j}(j=1, \ldots, n \geq 2)$ be $n$ linearly independent meromorphic functions. If $f_{1}+\ldots+f_{n} \equiv 1$, then we have

$$
\begin{aligned}
T\left(r, f_{1}\right) \leq & \sum_{j=1}^{n} N\left(r, \frac{1}{f_{j}}\right)+N(r, W)-N\left(r, \frac{1}{W}\right) \\
& -\sum_{j=2}^{n} N\left(r, f_{j}\right)+S\left(r, f_{1}\right)+\ldots+S\left(r, f_{n}\right),
\end{aligned}
$$

where $W=W(z)$ is the Wronskian of $f_{1}, \ldots, f_{n}$.

\section{Proof of Theorem 1.}

Let

$F_{1}=:\{z: a(z)=\infty\} \cup\{z: b(z)=\infty\} \cup\{z: \alpha(z)=\infty\} \cup\{z: \beta(z)=\infty\}$, $F_{2}=:\{z: a(z)=\alpha(z)\} \cup\{z: b(z)=\beta(z)\}$.

Set

$$
F=: F_{1} \cup F_{2},
$$

the corresponding counting function is denoted by $N_{F}(r)$. Put

$$
h(z)=: \quad \frac{f(z)-a(z)}{g(z)-b(z)} .
$$

Since $a(z)$ and $b(z)$ are small functions of $f$ and $g$ respectively, we know that $h(z) \not \equiv 0, \infty$. Let $z_{o}$ be a pole of $h$ with $z_{o} \notin F$. Since $f$ and $g$ share the 
pair $(a, b) \mathrm{CM}$, we have $z_{o} \in V_{2} \cup V_{3}$. If $z_{o} \in V_{2}$, then $\omega\left(h, z_{o}\right)=\omega\left(f, z_{o}\right)$; If $z_{o} \in V_{3}$, then $\omega\left(h, z_{o}\right)=\omega\left(f, z_{o}\right)-\omega\left(g, z_{o}\right)$. Thus

(9)

$N_{2}(f)+N_{3}(f)-N_{3}(g)-N_{F}(r) \leq N(r, h) \leq N_{2}(f)+N_{3}(f)-N_{3}(g)+N_{F}(r)$.

Similarly we have

$$
N\left(r, \frac{1}{h}\right) \leq N_{1}(g)+N_{5}(g)-N_{5}(f)+N_{F}(r) .
$$

Let

$$
f_{1}=: \frac{f-\alpha}{a-\alpha}, \quad f_{2}=:-\frac{g-\beta}{a-\alpha} h, \quad f_{3}=: \frac{b-\beta}{a-\alpha} h .
$$

Then

$$
\begin{gathered}
N\left(r, \frac{1}{f_{1}}\right) \leq N\left(r, \frac{1}{f-\alpha}\right)+N_{F}(r), \\
N\left(r, \frac{1}{f_{3}}\right) \leq N\left(r, \frac{1}{h}\right)+N_{F}(r), \\
N(r, h)-N_{F}(r) \leq N\left(r, f_{3}\right) \leq N(r, h)+N_{F}(r) .
\end{gathered}
$$

From (8) it is easy to see that any zero of $h$ which is not in the set $F$ is not a zero of $f_{2}$. Thus

$$
N\left(r, \frac{1}{f_{2}}\right) \leq N\left(r, \frac{1}{g-\beta}\right)+N_{F}(r) .
$$

Now for any pole $z_{o}$ of $f_{2}$ with $z_{o} \notin F$, we know that $z_{o}$ is a pole of $g$ or $h$. If $z_{o} \in V_{1}$, then $\omega\left(g, z_{o}\right)=\omega\left(\frac{1}{h}, z_{o}\right)$, and so, $\omega\left(f_{2}, z_{o}\right)=0$; If $z_{o} \in V_{2}$, then $\omega\left(f_{2}, z_{o}\right)=\omega\left(h, z_{o}\right)=\omega\left(f, z_{o}\right)$; If $z_{o} \in V_{3}$, then $\omega\left(h, z_{o}\right)=\omega\left(f, z_{o}\right)-\omega\left(g, z_{o}\right)$, and so, $\omega\left(f_{2}, z_{o}\right)=\omega\left(g, z_{o}\right)+\omega\left(h, z_{o}\right)=\omega\left(f, z_{o}\right)$; If $z_{o} \in V_{4}$, then $h\left(z_{o}\right) \neq 0, \infty$ and $\omega\left(f_{2}, z_{o}\right)=\omega\left(g, z_{o}\right)$; If $z_{o} \in V_{5}$, then $\omega\left(\frac{1}{h}, z_{o}\right)=\omega\left(g, z_{o}\right)-\omega\left(f, z_{o}\right)$ and $\omega\left(f_{2}, z_{o}\right)=\omega\left(g, z_{o}\right)-\omega\left(\frac{1}{h}, z_{o}\right)=\omega\left(f, z_{o}\right)$. Combining all these facts we get

$$
\begin{gathered}
N_{2}(f)+N_{3}(f)+N_{4}(g)+N_{5}(f)-N_{F}(r) \leq N\left(r, f_{2}\right) \\
\leq N_{2}(f)+N_{3}(f)+N_{4}(g)+N_{5}(f)+N_{F}(r) .
\end{gathered}
$$

Next we rewrite (8) in the form

$$
f_{1}+f_{2}+f_{3}=1 \text {. }
$$


Without loss of generality, we suppose that there exists a set $I$ with infinite measure such that

$$
T(r, g) \leq T(r, f), \quad r \in I .
$$

(Otherwise, we only need to consider $T(r, g)$ instead of $T(r, f)$ in the following discussions.) Thus $N_{F}(r)=S(r, f), r \in I$.

In the sequel, we always let $r \in I$. Now we prove the following lemma.

Lemma 2. $f_{1}, f_{2}$ and $f_{3}$ are linearly dependent.

Proof. Suppose on the contrary that the $f$ 's are linearly independent. By lemma 1,

$$
\begin{aligned}
T\left(r, f_{1}\right) \leq & \sum_{j=1}^{3} N\left(r, \frac{1}{f_{j}}\right)+N(r, W)-N\left(r, \frac{1}{W}\right)-N\left(r, f_{2}\right)-N\left(r, f_{3}\right) \\
& +S\left(r, f_{1}\right)+S\left(r, f_{2}\right)+S\left(r, f_{3}\right)
\end{aligned}
$$

where $W$ is the Wronskian of $f_{1}, f_{2}, f_{3}$, i.e.,

$$
W=\left|\begin{array}{lll}
f_{1} & f_{2} & f_{3} \\
f_{1}^{\prime} & f_{2}^{\prime} & f_{3}^{\prime} \\
f_{1}^{\prime \prime} & f_{2}^{\prime \prime} & f_{3}^{\prime \prime}
\end{array}\right|=-\left|\begin{array}{ll}
f_{1}^{\prime} & f_{3}^{\prime} \\
f_{1}^{\prime \prime} & f_{3}^{\prime \prime}
\end{array}\right|
$$

by (16). Now by (10), (11), (12) and (14),

$$
\begin{aligned}
\sum_{j=1}^{3} N\left(r, \frac{1}{f_{j}}\right) \leq & N\left(r, \frac{1}{f-\alpha}\right)+N\left(r, \frac{1}{g-\beta}\right) \\
& +N_{1}(g)+N_{5}(g)-N_{5}(f)+4 N_{F}(r) .
\end{aligned}
$$

In addition, by the inequalities on the left hand sides of (9), (13) and (15), $N\left(r, f_{2}\right)+N\left(r, f_{3}\right) \geq 2 N_{2}(f)+2 N_{3}(f)+N_{4}(f)+N_{5}(f)-N_{3}(g)-3 N_{F}(r)$.

Combining the three inequalities above we get

$$
\begin{aligned}
T\left(r, f_{1}\right) \leq & N(r, W)-N\left(r, \frac{1}{W}\right)+N\left(r, \frac{1}{f-\alpha}\right)+N\left(r, \frac{1}{g-\beta}\right) \\
& +N_{1}(g)+N_{3}(g)+N_{5}(g) \\
& -2 N_{2}(f)-2 N_{3}(f)-N_{4}(f)-2 N_{5}(f) \\
& +7 N_{F}(r)+S\left(r, f_{1}\right)+S\left(r, f_{2}\right)+S\left(r, f_{3}\right)
\end{aligned}
$$




$$
\begin{aligned}
= & N(r, W)-N\left(r, \frac{1}{W}\right)+N\left(r, \frac{1}{f-\alpha}\right)+N\left(r, \frac{1}{g-\beta}\right) \\
& +N_{1}(g)+N_{3}(g)+N_{4}(g)+N_{5}(g) \\
& -2 N_{2}(f)-2 N_{3}(f)-2 N_{4}(f)-2 N_{5}(f) \\
& -N_{4}(g)+N_{4}(f) \\
& +7 N_{F}(r)+S\left(r, f_{1}\right)+S\left(r, f_{2}\right)+S\left(r, f_{3}\right) .
\end{aligned}
$$

Substituting (6) and (7) into the above inequality and using the facts that

$$
\begin{gathered}
N_{4}(f)=N_{4}(g), \quad T(r, f)=T\left(r, f_{1}\right)+S(r, f), \\
T(r, a), \quad T(r, b), \quad T(r, \alpha), \quad T(r, \beta)=S(r, f), \\
N_{F}(r), \quad S\left(r, f_{j}\right)=S(r, f), \quad(j=1, \ldots, 3)
\end{gathered}
$$

we obtain

$$
\begin{gathered}
T(r, f) \leq N\left(r, \frac{1}{f-\alpha}\right)+N\left(r, \frac{1}{g-\beta}\right)+N(r, W)-N\left(r, \frac{1}{W}\right) \\
+N(r, g)-2 N(r, f)+S(r, f) .
\end{gathered}
$$

Next we estimate the term $N(r, W)-N\left(r, \frac{1}{W}\right)$. Since

$$
W=-\left(f_{1}^{\prime} f_{3}^{\prime \prime}-f_{1}^{\prime \prime} f_{3}^{\prime}\right)
$$

from the expressions of $f_{1}$ and $f_{3}$ we see that the poles of $W$ only occur at the poles of $f$ and the points in $F$. Let $z_{0}$ be a pole of $f$ with $z_{0} \notin F$.

If $z_{o} \in V_{2}$, then near $z=z_{0}$,

$f_{1}=\frac{1}{\left(z-z_{0}\right)^{\omega\left(f, z_{0}\right)}}\left\{x+\bigcirc\left(z-z_{0}\right)\right\}, \quad f_{3}=\frac{1}{\left(z-z_{0}\right)^{\omega\left(f, z_{0}\right)}}\left\{y+\bigcirc\left(z-z_{0}\right)\right\}$

where $x$ and $y$ are nonzero constants. If $\omega\left(f, z_{0}\right) \geq 2$, then

$$
\begin{aligned}
& f_{1}^{\prime} f_{3}^{\prime \prime}=\frac{1}{\left(z-z_{0}\right)^{2 \omega\left(f, z_{0}\right)+3}}\left\{-\omega\left(f, z_{0}\right)^{2}\left(\omega\left(f, z_{0}\right)+1\right) x y+\bigcirc\left(z-z_{0}\right)\right\}, \\
& f_{1}^{\prime \prime} f_{3}^{\prime}=\frac{1}{\left(z-z_{0}\right)^{2 \omega\left(f, z_{0}\right)+3}}\left\{-\omega\left(f, z_{0}\right)^{2}\left(\omega\left(f, z_{0}\right)+1\right) x y+\bigcirc\left(z-z_{0}\right)\right\},
\end{aligned}
$$

and so,

$$
f_{1}^{\prime} f_{3}^{\prime \prime}-f_{1}^{\prime \prime} f_{3}^{\prime}=\bigcirc\left\{\frac{1}{\left(z-z_{0}\right)^{2 \omega\left(f, z_{0}\right)+2}}\right\}
$$


If $\omega\left(f, z_{0}\right)=1$, then

$$
\begin{aligned}
& f_{1}^{\prime} f_{3}^{\prime \prime}=\frac{-2 x y}{\left(z-z_{0}\right)^{5}}+\frac{\bigcirc(1)}{\left(z-z_{0}\right)^{3}}+\ldots \\
& f_{1}^{\prime \prime} f_{3}^{\prime}=\frac{-2 x y}{\left(z-z_{0}\right)^{5}}+\frac{\bigcirc(1)}{\left(z-z_{0}\right)^{3}}+\ldots
\end{aligned}
$$

and so,

$$
f_{1}^{\prime} f_{3}^{\prime \prime}-f_{1}^{\prime \prime} f_{3}^{\prime}=\bigcirc\left\{\frac{1}{\left(z-z_{0}\right)^{3}}\right\}
$$

Thus

$$
\begin{gathered}
\omega\left(W, z_{0}\right) \leq \begin{cases}2 \omega\left(f, z_{0}\right)+2, & \text { if } \omega\left(f, z_{0}\right) \geq 2 \\
3, & \text { if } \omega\left(f, z_{0}\right)=1\end{cases} \\
\quad \leq 3 \omega\left(f, z_{0}\right) .
\end{gathered}
$$

If $z_{0} \in V_{3}$, then $\omega\left(g, z_{0}\right) \geq 1$ and $\omega\left(f, z_{0}\right) \geq 2$. Thus, by (19),

$$
\begin{gathered}
\omega\left(W, z_{0}\right) \leq 2 \omega\left(f, z_{0}\right)+3-\omega\left(g, z_{0}\right) \\
\leq 2 \omega\left(f, z_{0}\right)+2 \leq 3 \omega\left(f, z_{0}\right) .
\end{gathered}
$$

If $z_{0} \in V_{4}$, then $\omega\left(f, z_{0}\right)=\omega\left(g, z_{0}\right)$, and so, $\omega\left(f_{3}, z_{0}\right)=0$. By (19), we get

$$
\omega\left(W, z_{0}\right) \leq \omega\left(f, z_{0}\right)+2 \leq 3 \omega\left(f, z_{0}\right) .
$$

If $z_{0} \in V_{5}$, and if $z_{0}$ is a pole of $W$, then by (19),

$$
\begin{gathered}
\omega\left(W, z_{0}\right) \leq \omega\left(f, z_{0}\right)+2 \\
\leq 3 \omega\left(f, z_{0}\right) .
\end{gathered}
$$

Combining all the cases above and noting (6), we deduce that

$$
N(r, W) \leq 3 N(r, f)+N_{F}(r) .
$$

This and (18) give

(20) $T(r, f) \leq N\left(r, \frac{1}{f-\alpha}\right)+N\left(r, \frac{1}{g-\beta}\right)+N(r, f)+N(r, g)+S(r, f)$.

Now by the definition of deficiency, for $\epsilon=\frac{\delta-3}{8}>0$, where $\delta$ is the sum in (3), there exists $r_{o}>0$ such that

$$
N\left(r, \frac{1}{f-\alpha}\right) \leq(1-\delta(\alpha, f)+\epsilon) T(r, f),
$$




$$
\begin{gathered}
N\left(r, \frac{1}{g-\beta}\right) \leq(1-\delta(\beta, g)+\epsilon) T(r, g), \\
N(r, f) \leq(1-\delta(\infty, f)+\epsilon) T(r, f)
\end{gathered}
$$

and

$$
N(r, g) \leq(1-\delta(\infty, g)+\epsilon) T(r, g)
$$

hold for $r \in I$ and $r>r_{o}$. Substituting all these inequality into (20) and noting (17), we get $\delta \leq 3$, which contradicts our hypothesis. This completes the proof of the lemma.

Now by Lemma 2, there exist three constants $c_{1}, c_{2}$ and $c_{3}$ with

$$
\left|c_{1}\right|+\left|c_{2}\right|+\left|c_{3}\right| \neq 0
$$

and

$$
c_{1} f_{1}+c_{2} f_{2}+c_{3} f_{3}=0 .
$$

If $c_{1}=0$, then $c_{2} c_{3} \neq 0$ and $f_{2}=-\frac{c_{3}}{c_{2}} f_{3}$. This leads to $g=\frac{c_{3}}{c_{2}} b(z)+$ $\left(1-\frac{c_{3}}{c_{2}}\right) \beta(z)$, which contradicts the assumptions that $b(z)$ and $\beta(z)$ are small functions of $g$. Thus, $c_{1} \neq 0$. We may suppose $c_{1}=-1$, and (22) reads $f_{1}=c_{2} f_{2}+c_{3} f_{3}$. Combining this and (16) we obtain

$$
\left(1+c_{2}\right) f_{2}+\left(1+c_{3}\right) f_{3}=1 \text {. }
$$

Next we consider two cases.

(i) $1+c_{2}=0$. Then $1+c_{3} \neq 0$ and $\left(1+c_{3}\right) f_{3}=1$. It follows from (8) and the definition of $f_{3}$ between (10) and (11) that

$$
\begin{aligned}
f-\frac{c_{3} a+\alpha}{1+c_{3}} & =f-a+a-\frac{c_{3} a+\alpha}{1+c_{3}} \\
& =\left(\frac{1}{1+c_{3}}\right) \frac{a-\alpha}{b-\beta}(g-b)+a-\frac{c_{3} a+\alpha}{1+c_{3}} \\
& =\left(\frac{1}{1+c_{3}}\right) \frac{a-\alpha}{b-\beta}(g-\beta) .
\end{aligned}
$$

If $c_{3} \neq 0$, then $\frac{c_{3} a+\alpha}{1+c_{3}} \neq \alpha$. By the Nevanlinna "three-functions theorem" we deduce that

$$
\begin{aligned}
T(r, f) & \leq N(r, f)+N\left(r, \frac{1}{f-\alpha}\right)+N\left(r, \frac{1}{f-\frac{c_{3} a+\alpha}{1+c_{3}}}\right)+S(r, f) \\
= & N(r, f)+N\left(r, \frac{1}{f-\alpha}\right)+N\left(r, \frac{1}{g-\beta}\right)+S(r, f) .
\end{aligned}
$$


This is impossible by the same reasoning as in the proof of Lemma 2. Therefore $c_{3}=0$ and (24) reads

$$
\frac{f-\alpha}{g-\beta}=\frac{a-\alpha}{b-\beta}
$$

This is what we need.

(ii) $1+c_{2} \neq 0$. It follows from (8), (23) and the definitions of $f_{2}$ and $f_{3}$ between (10) and (11) that $-\left(1+c_{2}\right) \frac{g-\beta}{a-\alpha}+\left(1+c_{3}\right) \frac{b-\beta}{a-\alpha}=\frac{g-b}{f-a}$, which can be written as

$$
f-\frac{c_{2} a+\alpha}{1+c_{2}}=\left(\frac{c_{2}-c_{3}}{\left(1+c_{2}\right)^{2}}\right) \frac{(a-\alpha)(b-\beta)}{g-\frac{1+c_{3}}{1+c_{2}} b-\frac{c_{2}-c_{3}}{1+c_{2}} \beta} .
$$

If $c_{2} \neq 0$, then $\frac{c_{2} a+\alpha}{1+c_{2}} \neq \alpha$. By the "three-functions theorem", we have

$$
\begin{aligned}
T(r, f) \leq & N(r, f)+N\left(r, \frac{1}{f-\alpha}\right)+N\left(r, \frac{1}{f-\frac{c_{2} a+\alpha}{1+c_{2}}}\right)+S(r, f) \\
& \leq N(r, f)+N\left(r, \frac{1}{f-\alpha}\right)+N(r, g)+S(r, f) .
\end{aligned}
$$

By the same reasoning as in the proof of Lemma 2, we can get a contradiction. Thus $c_{2}=0$, and (25) reads

$$
f-\alpha=-c_{3} \frac{(a-\alpha)(b-\beta)}{g-\left(1+c_{3}\right) b+c_{3} \beta} .
$$

If $c_{3}=-1$, then

$$
(f-\alpha)(g-\beta)=(a-\alpha)(b-\beta),
$$

as asserted. If $c_{3} \neq-1$, then $\frac{\alpha+c_{3} a}{1+c_{3}} \neq \alpha$ and (26) can be written as

$$
f-\frac{\alpha+c_{3} a}{1+c_{3}}=-\left(\frac{c_{3}}{1+c_{3}}\right) \frac{(a-\alpha)(g-\beta)}{g-\left(1+c_{3}\right) b+c_{3} \beta} .
$$

Thus, the "three-functions theorem" gives

$$
\begin{aligned}
T(r, f) & \leq N(r, f)+N\left(r, \frac{1}{f-\alpha}\right)+N\left(r, \frac{1}{f-\frac{\alpha+c_{3} a}{1+c_{3}}}\right)+S(r, f) \\
& \leq N(r, f)+N\left(r, \frac{1}{f-\alpha}\right)+N\left(r, \frac{1}{g-\beta}\right)+S(r, f) .
\end{aligned}
$$

By the same reasoning as in the proof of Lemma 2 we obtain a contradiction. This completes the proof of the theorem. 
Acknowledgement. I wish to thank Prof. Dr. G. Frank, Prof. Dr. E. Mues and Dr. M. Reinders for their valuable discussions. I also want to express my gratitude to the refree for many useful suggestions.

\section{References}

[1] C.T. Chuang, Une généralisation d'une inegatité de Nevanlinna, Sci. Sinica, 13 (1964), 887-895.

[2] C.T. Chuang and X.H. Hua, On the growth of meromorphic functions, Sci. Sinica (Sci. in China), 33 (1990), 1025-1033.

[3] C.T. Chuang and C.C. Yang, Fix-points and factorization of meromorphic functions, World Sci. Publishing, Singapore 1990.

[4] G. Frank and W. Ohlenroth, Meromorphe Funktionen, die mit einer ihrer Ableitungen Werte teilen, Complex Variables, 6 (1986), 23-27.

[5] F. Gross, Factorization of meromorphic functions, U. S. Goverment Printing Office 1972.

[6] G.G. Gundersen, Meromorphic functions that share three or four values, J. London Math. Soc., 20 (1979), 457-466.

[7] M. Ozawa, Unicity theorems for entire functions, J. Analyse Math., 30 (1976), 411-420.

[8] H. Ueda, Unicity theorems for entire functions, Kodai Math. J., 3 (1980), 212-223.

[9] C.C. Yang, On meromorphic functions taking the same values at the same points, Kodai Math Sem. Rep., 28 (1977), 300-309.

[10] H.X. Yi, Meromorphic functions with two deficient values, Acta Math. Sinica, 30 (1987), 588-597.

Received November 10, 1993 and revised October 4, 1994. Supported by DAAD/K.C.Wong Fellowship and NNSF of China.

NANJING UNIVERSITY

NANJING 210093

P.R.CHINA

E-mail address: postmath@netra.nju.edu.cn 



\title{
PACIFIC JOURNAL OF MATHEMATICS
}

\author{
Founded in 1951 by
}

\author{
E. F. Beckenbach (1906-1982) $\quad$ F. Wolf (1904-1989)
}

\section{EDITORS}

Sun-Yung A. Chang (Managing Editor) Robert Finn University of California

Los Angeles, CA 90095-1555

pacific@math.ucla.edu

\section{F. Michael Christ}

University of California

Los Angeles, CA 90095-1555

christ@math.ucla.edu

Nicholas Ercolani

University of Arizona

Tucson, AZ 85721

ercolani@math.arizona.edu
Stanford University

Stanford, CA 94305

finn@gauss.stanford.edu

Steven Kerckhoff

Stanford University

Stanford, CA 94305

spk@gauss.stanford.edu

Martin Scharlemann

University of California

Santa Barbara, CA 93106

mgscharl@math.ucsb.edu

\section{Gang Tian}

Massachusettes Institute of Technology

Cambridge, MA 02139

tian@math.mit.edu

\section{V.S. Varadarajan}

University of California

Los Angeles, CA 90095-1555

vsv@math.ucla.edu

Dan Voiculescu

University of California

Berkeley, CA 94720

dvv@math.berkeley.edu

\section{SUPPORTING INSTITUTIONS}

CALIF. INST. OF TECHNOLOGY CHINESE UNIV. OF HONG KONG MACQUARIE UNIVERSITY NEW MEXICO STATE UNIV. OREGON STATE UNIV. PEKING UNIVERSITY RITSUMEIKAN UNIVERSITY STANFORD UNIVERSITY TOKYO INSTITUTE OF TECHNOLOGY UNIVERSIDAD DE LOS ANDES
UNIV. OF ARIZONA

UNIV. OF BRITISH COLUMBIA

UNIV. OF CALIF., BERKELEY

UNIV. OF CALIF., DAVIS

UNIV. OF CALIF,, IRVINE UNIV. OF CALIF., LOS ANGELES

UNIV. OF CALIF., RIVERSIDE UNIV. OF CALIF., SAN DIEGO UNIV. OF CALIF., SANTA BARBARA UNIV. OF CALIF., SANTA CRUZ
UNIV. OF HAWAII

UNIV. OF MELBOURNE

UNIV. OF MONTANA

UNIV. NACIONAL AUTONOMA DE MEXICO

UNIV. OF NEVADA, RENO

UNIV. OF OREGON

UNIV. OF SOUTHERN CALIFORNIA

UNIV OF UTAH

UNIV. OF WASHINGTON

WASHINGTON STATE UNIVERSITY

The supporting Institutions listed above contribute to the cost of publication of this Journal, but they are not owners or publishers and have no responsibility for its contents or policies.

Manuscripts must be prepared in accordance with the instructions provided on the inside back cover.

The table of contents and the abstracts of the papers in the current issue, as well as other information about the Pacific Journal of Mathematics, may be found on the Internet at http://www.math.uci.edu/pjm.html.

The Pacific Journal of Mathematics (ISSN 0030-8730) is published monthly except for July and August. Regular subscription rate: $\$ 245.00$ a year (10 issues). Special rate: $\$ 123.00$ a year to individual members of supporting institutions.

Subscriptions, back issues published within the last three years and changes of subscribers address should be sent to Pacific Journal of Mathematics, P.O. Box 4163, Berkeley, CA 94704-0163, U.S.A. Prior back issues are obtainable from Kraus Periodicals Co., Route 100, Millwood, NY 10546.

The Pacific Journal of Mathematics at the University of California, c/o Department of Mathematics, 981 Evans Hall, Berkeley, CA 94720 (ISSN 0030-8730) is published monthly except for July and August. Second-class postage paid at Berkeley, CA 94704, and additional mailing offices. POSTMASTER: send address changes to Pacific Journal of Mathematics, P.O. Box 6143, Berkeley, CA 94704-0163.

PUBLISHED BY PACIFIC JOURNAL OF MATHEMATICS at University of California, Berkeley, CA 94720, A NON-PROFIT CORPORATION

This publication was typeset using AMS-LATEX,

the American Mathematical Society's TEX macro system.

Copyright (c) 1995 by Pacific Journal of Mathematics 


\title{
PACIFIC JOURNAL OF MATHEMATICS
}

\author{
Volume $175 \quad$ No. $1 \quad$ September 1996
}

Homogeneous Ricci positive 5-manifolds

Dimitri Alekseevsky, Isabel Dotti de Miatello and CARlos J.

FERRARIS

On the structure of tensor products of $\ell_{p}$-spaces

ALVARO ARIAS and JEFFREY D. FARMER

The closed geodesic problem for compact Riemannian 2-orbifolds

JosEPH E. BORZELLINO and BENJAMIN G. LORICA

Small eigenvalue variation and real rank zero

Ola Bratteli and George A. Elliott

Global analytic hypoellipticity of $\square_{b}$ on circular domains

So-CHIN CHEN

Sharing values and a problem due to C. C. Yang

XIN-Hou HuA

Commutators and invariant domains for Schrödinger propagators

Min-JEI HUANG

Chaos of continuum-wise expansive homeomorphisms and dynamical properties of sensitive maps of graphs

\section{HiS AO KATO}

Some properties of Fano manifolds that are zeros of sections in homogeneous vector bundles over Grassmannians

\section{OLIVER KÜCHLE}

On polynomials orthogonal with respect to Sobolev inner product on the unit circle

XIN Li and FRANCISCO MARCELLAN

Maximal subfields of $\mathbf{Q}(i)$-division rings

STEVEN LIEDAHL

Virtual diagonals and $n$-amenability for Banach algebras

AlAN L. T. PATERSON

Rational Pontryagin classes, local representations, and $K^{G}$-theory

Claude Schochet

An equivalence relation for codimension one foliations of 3-manifolds

SANDRA SHIELDS

A construction of Lomonosov functions and applications to the invariant subspace problem

\section{ALEKSANDER SIMONIČ}

Complete intersection subvarieties of general hypersurfaces 